Fundación

Miguel Lillo

Tucumán

Argentina

\title{
Primeros registros de ocelote Leopardus pardalis (Linneaus, 1758) (Carnivora: Felidae) en la provincia de Catamarca, Argentina
}

First records of the ocelot Leopardus pardalis (Linneaus, 1758) (Carnivora: Felidae) from Catamarca province, Argentina

\section{Gonzalo Martínez}

Departamento de Biología, Facultad de Ciencias Exactas y Naturales, Universidad Nacional de Catamarca (UNCA). Av. Belgrano 300, (4700) San Fernando del Valle de Catamarca, Argentina.

Autor corresponsal: gonza.catamarca@gmail.com

\section{RESUMEN}

Leopardus pardalis tiene una amplia distribución en la región Neotropical y su estado de conservación es de preocupación menor a nivel mundial. Sin embargo, en Argentina actualmente se encuentra en estado vulnerable debido a las actividades humanas, como la cacería por su piel y el desmonte. En base a las más recientes publicaciones sobre la distribución de esta especie, la provincia de Catamarca está incluida como sitio probable de presencia en base a registros no documentados de pobladores locales. El presente estudio confirma la presencia actual de L. pardalis en la provincia de Catamarca mediante registros fotográficos con cámara trampa. Con este primer reporte de $L$. pardalis en Catamarca se pueden tomar medidas urgentes para la conservación de su hábitat.

Palabras clave - Primer reporte, ocelote, gato onza, Yungas.

\section{ABSTRACT}

Leopardus pardalis has a wide distribution in the Neotropical regions and its conservation status is of lesser concern worldwide. However, in Argentina it has a vulnerable state due to human activities such as hunting for their fur and deforestation. Based on the most recent publications about the distribution of this species, Catamarca is included as a possible site of presence, based on undocumented records of local people. The present study confirms the current presence of L. pardalis in the

\footnotetext{
Ref. bibliográfica: G. Martínez. 2021. "Primeros registros de ocelote Leopardus pardalis (Linneaus, 1758) (Carnivora: Felidae) en la provincia de Catamarca, Argentina". Acta zoológica lilloana 65 (1): 1-9. doi: https:// doi.org/10.30550/j.azl/2021.65.1/2021-02-11

> Recibido: 23 de julio 2020 - Aceptado: 11 de febrero 2021.

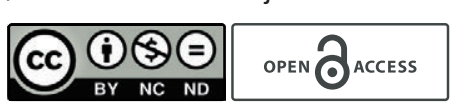

- URL de la revista: http://actazoologica.lillo.org.ar

- Esta obra está bajo una Licencia Creative Commons Atribución - No Comercial - Sin Obra Derivada 4.0 Internacional.
} 
province of Catamarca through photographic records with a camera trap. With this record, urgent measures can be taken to preserve its habitat.

Keywords - First report, ocelot, Yungas, NOA.

Leopardus pardalis (Linneaus, 1758), conocida vulgarmente como ocelote o gato onza, es un felino de tamaño mediano, con hábitos principalmente nocturnos y terrestres. Habita diversos ecosistemas en el continente americano dentro de la región Neotropical, desde el sur de los Estados Unidos hasta el norte de Argentina (Clavijo y Ramírez, 2009). En este último parece estar asociado a bosques y selvas en buen estado de conservación, principalmente en la Selva Paranaense, donde el Parque Nacional Iguazú tiene la mayor cantidad de registros (Sistema de Información de Biodiversidad [SIB] (2020)). Sin embargo, existen reportes documentados en las provincias de Corrientes, Formosa, Chaco, Jujuy, Salta y Tucumán, con dudas en Santiago del Estero y norte de Santa Fe en cuanto a su distribución actual (Canevari y Fernández Balboa, 2003; Chatellenaz, Villordo, Anchetti y Rolón, 2018; Parera, 2018). En la provincia de Catamarca no existen datos históricos de la presencia de $L$. pardalis (Cabrera, 1957; Chebez, 1994) y hasta el año 2018 tampoco era considerada como una especie de presencia dudosa o probable para la provincia. Sin embargo, Cruz et al. (2019) realizan la primera mención de la probable presencia de $L$. pardalis en Catamarca en base a comunicaciones personales de pobladores.

El estado de conservación a nivel global es de Preocupación Menor y la tendencia poblacional es decreciente según la última evaluación hecha por la Unión Internacional para la Conservación de la Naturaleza (IUCN) (Paviolo et al., 2016). Sin embargo, en Argentina es considerada como una especie Vulnerable según la Sociedad Argentina para el Estudio de los Mamíferos (SAREM) (Cruz et al., 2019). De hecho, se estima que las poblaciones de esta especie habrían declinado un $30 \%$ en los últimos 25 años a causa de varios factores, como la persecución y cacería por su piel o por la reducción notable de su hábitat producto de los desmontes para el avance de la frontera agrícola (Cruz et al., 2019). Estos factores de perturbación son de particular relevancia en las regiones del Chaco y las Yungas (Brown, Martínez Ortiz, Acerbi y Corcuera, 2006; Nanni et al., 2020).

El objetivo de este trabajo es dar a conocer los primeros registros documentados de L. pardalis en la provincia de Catamarca.

La zona donde se efectuaron los registros se conoce como Quebrada de Los Laureles, en el departamento Paclín, a 9 kilómetros hacia el noreste de la localidad de La Merced (Fig. 1). Se trata de un área ubicada en el distrito de Selva Montana de la región fitogeográfica de las Yungas, que se encuentra en muy buen estado de conservación (Perea, Pedraza y Luceros, 2006) pese a la actividad ganadera y a la presencia del intenso tránsito que circula por la Cuesta del Totoral (Ruta Nacional 38). La estructura selvática está compuesta por diferentes pisos altitudinales. Entre los árboles emergentes del dosel están Blepharocalyx salicifolius, Cedrela angustifolia y Ocotea porphyria, mientras que el piso inferior está compuesto por especies arbóreas 


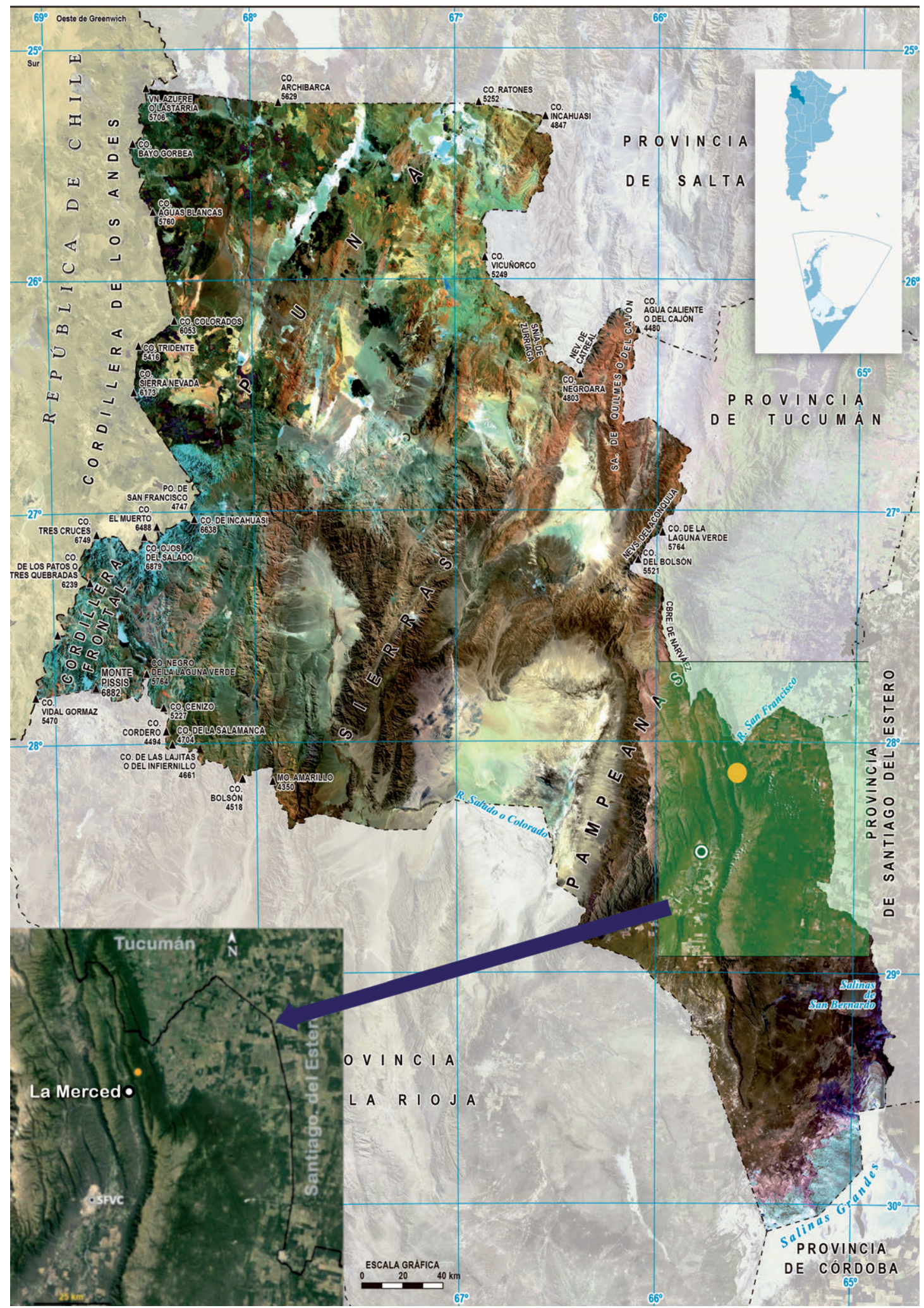

Figura 1. Ubicación geográfica del registro documentado de L. pardalis en la provincia de Catamarca. El punto amarillo representa el lugar de los registros. Mapa base satelital extraído del Instituto Geográfico Nacional [IGN] (2014) y mapa agregado extraído de Google Earth (2019).

Figure 1. Geographic location of the documented record of L. pardalis in Catamarca province. The yellow dot represents the site of the records. Satellite base map extracted from the Instituto Nacional de Geografía [IGN] (2014) and aggregated map extracted from Google Earth (2019). 
como Parapiptadenia excelsa, Fuglans australis, Myrcianthes pungens, Myrrhinium atropurpureum, entre otros. La estructura arbustiva y el sotobosque son muy densos, con una gran cantidad de helechos, lianas, musgos, etc. (Morlans, 1995; Perea et al., 2006).

Para la obtención de los registros se colocó una sola cámara trampa de marca Scatolly HC- $300 M^{\odot}$ en las inmediaciones del arroyo Los Laureles $\left(28^{\circ} 05^{\prime} 57^{\prime}\right.$ 'S, $65^{\circ} 37^{\prime} 12^{\prime}$ W), al borde de un sendero de animales que atraviesa la selva paralelamente al arroyo, a una altitud de 1068 m s.n.m. Se realizaron dos campañas de muestreo con el método de fototrampeo en el lugar descrito. El 26 de agosto de 2019 se colocó la cámara trampa en este sitio por primera vez y sin la asistencia de cebo específico para la atracción de carnívoros. Durante este muestreo la cámara trampa estuvo activa apenas cinco días debido a la gran actividad de avifauna presente en el lugar, principalmente de Cyanocorax chrysops, especie que activó el sensor de movimiento una gran cantidad de veces. La cámara se programó para efectuar tres disparos fotográficos en intervalos de 5 segundos y luego un video de 30 segundos. Un año después se colocó la misma cámara a tan solo 100 metros del sitio anterior, el día 22 de agosto de 2020. Allí estuvo activa solamente cuatro días, también por causa de la gran actividad de $C$. chrysops. En esta ocasión, al frente de la cámara se colocó como cebo una pequeña cantidad de atún para la atracción de carnívoros. Esta vez la cámara se programó para efectuar un solo disparo fotográfico y luego de 5 segundos un video cuya duración es de 30 segundos. En ambas ocasiones la cámara trampa se situó a 1 metro del suelo y no se utilizó ningún elemento específico para medir el tamaño de los animales. Se eligió el mes de agosto en ambas campañas para colocar el dispositivo ya que al ser el mes más seco del año (Servicio Meteorológico Nacional [SMN] (2020)) el sotobosque se encuentra despejado de vegetación y existe menor dificultad para transitar por los senderos escarpados del lugar al no haber lluvias. Teniendo en cuenta que este es un estudio preliminar para conocer la presenciaausencia de la especie no se tuvieron en cuenta métodos para la diferenciación de los individuos.

Se obtuvieron dos registros documentados de L. pardalis en diferentes fechas, con diferencia de un año. El primer registro se produjo el día 30 de agosto de 2019 a las 5:38 hs (Fig. 2). El individuo apareció solo en la primera fotografía y sin registro alguno de video. Si bien la mala calidad de la fotografía sumada a la sobreexposición lumínica y a la posición del individuo captado hacen que sea dificultosa la identificación de la especie, se logra observar el patrón de manchas característico de su cuerpo, que lo diferencia de las otras especies del género Leopardus spp. citadas para el NOA. Sin embargo, para confirmar fehacientemente su identificación se arribó posteriormente al lugar del registro y con cinta métrica se tomaron medidas del arbusto que aparece justo al lado del individuo en la foto, pudiendo estimar así la altura del felino, que ronda $10550 \mathrm{~cm}$ a la cabeza.

El segundo registro se obtuvo el día 23 de agosto de 2020 a las 21:09 hs. En esta ocasión, el individuo registrado activó la cámara tres veces, llegando a aparecer en dos fotos y en tres videos (Fig. 3 y 4). Estas imágenes son más claras y contundentes para la identificación de la especie. Además del patrón de sus manchas, se distingue claramente que su cola es muy corta y ancha en relación con el cuerpo, otra característica diferenciable de los felinos de tamaño pequeño (Chebez, 1994; Canevari y 


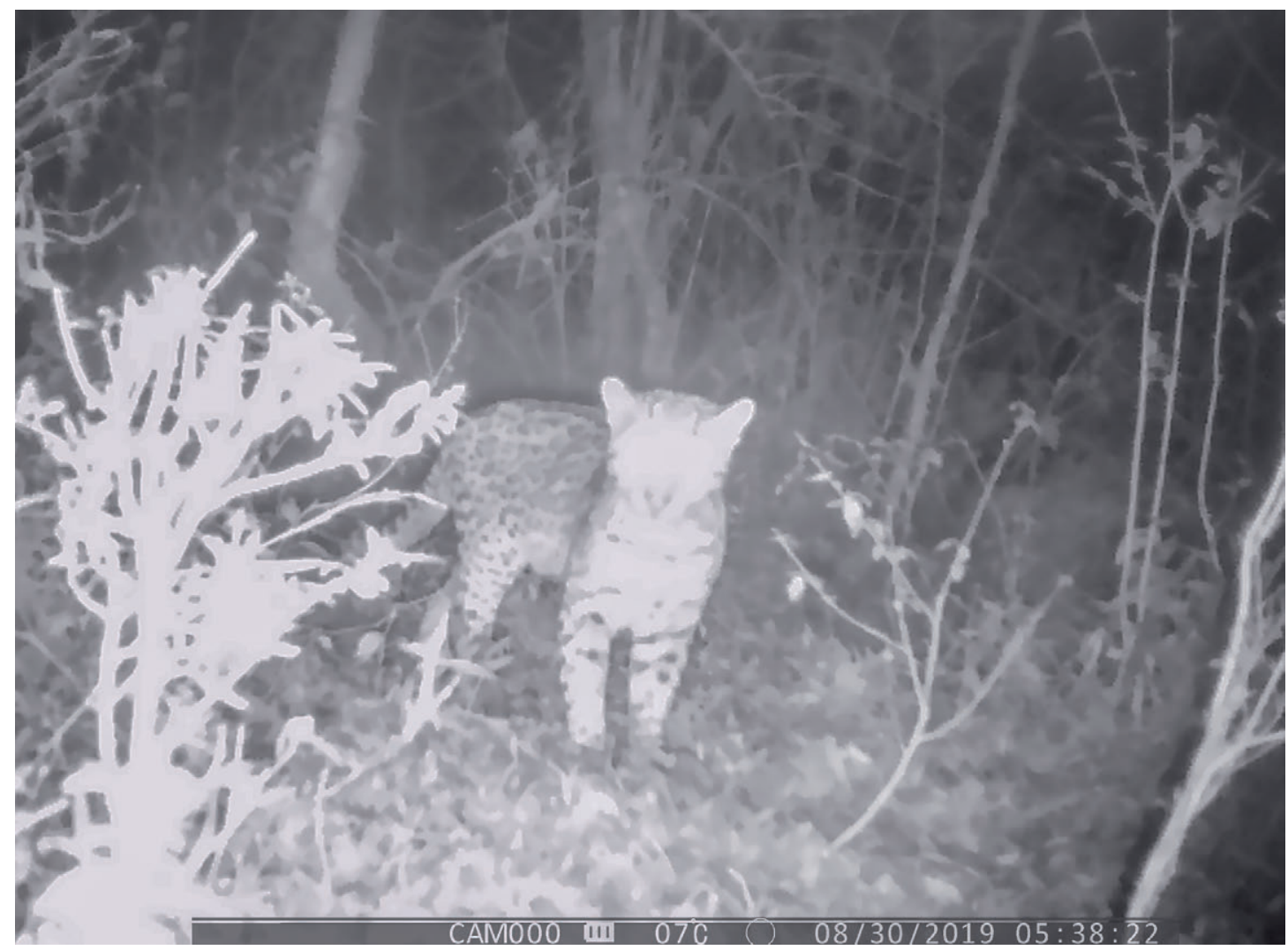

Figura 2. Individuo de L. pardalis registrado en las cercanías del arroyo Los Laureles, departamento Paclín, provincia de Catamarca, el día 30 de agosto de 2019.

Figure 2. Specimen of $L$. pardalis registered near the Los Laureles stream, Paclín department, Catamarca province, on August 30, 2019.

Fernández Balboa, 2003). En los videos se lo pudo observar olfateando todo el sector en busca del cebo colocado para su atracción (Fig. 4).

En conclusión, la información que brinda este trabajo confirma la presencia actual de $L$. pardalis en la provincia de Catamarca. Teniendo en cuenta que fue registrado en dos años consecutivos, en el mismo sitio y con una sola cámara trampa que estuvo activa apenas 4 o 5 días, se puede inferir que esta zona de Catamarca es un lugar de residencia para este felino y probablemente se trate de una especie común para la zona. No obstante, en estudios futuros y con mayor esfuerzo de muestreo (mayor cantidad de días y de cámaras activas, e idealmente apareadas y enfrentadas), se podrá determinar si se trata del mismo individuo o no y si existe una población reproductiva en el lugar. Hasta el momento, las Yungas catamarqueñas en buen estado de conservación no habían sido estudiadas mediante la técnica de fototrampeo y las citas de esta especie muestran la presencia actual e histórica de este felino solamente hasta la provincia de Tucumán (Cavenari y Fernández Balboa, 2003; Barquez, Díaz y Ojeda, 2006; Parera, 2018; APN, 2020), a excepción de Cruz et al. (2019), quienes a través de SAREM consideraron la presencia dudosa o probable de $L$. pardalis en Catamarca. También existen estudios de hace más de 17 años que determinaron la presencia documentada de L. pardalis en la provincia de Santiago del Estero (Jayat, Barquez, Díaz y Martínez, 1999; Caziani et al., 2003), aunque en 


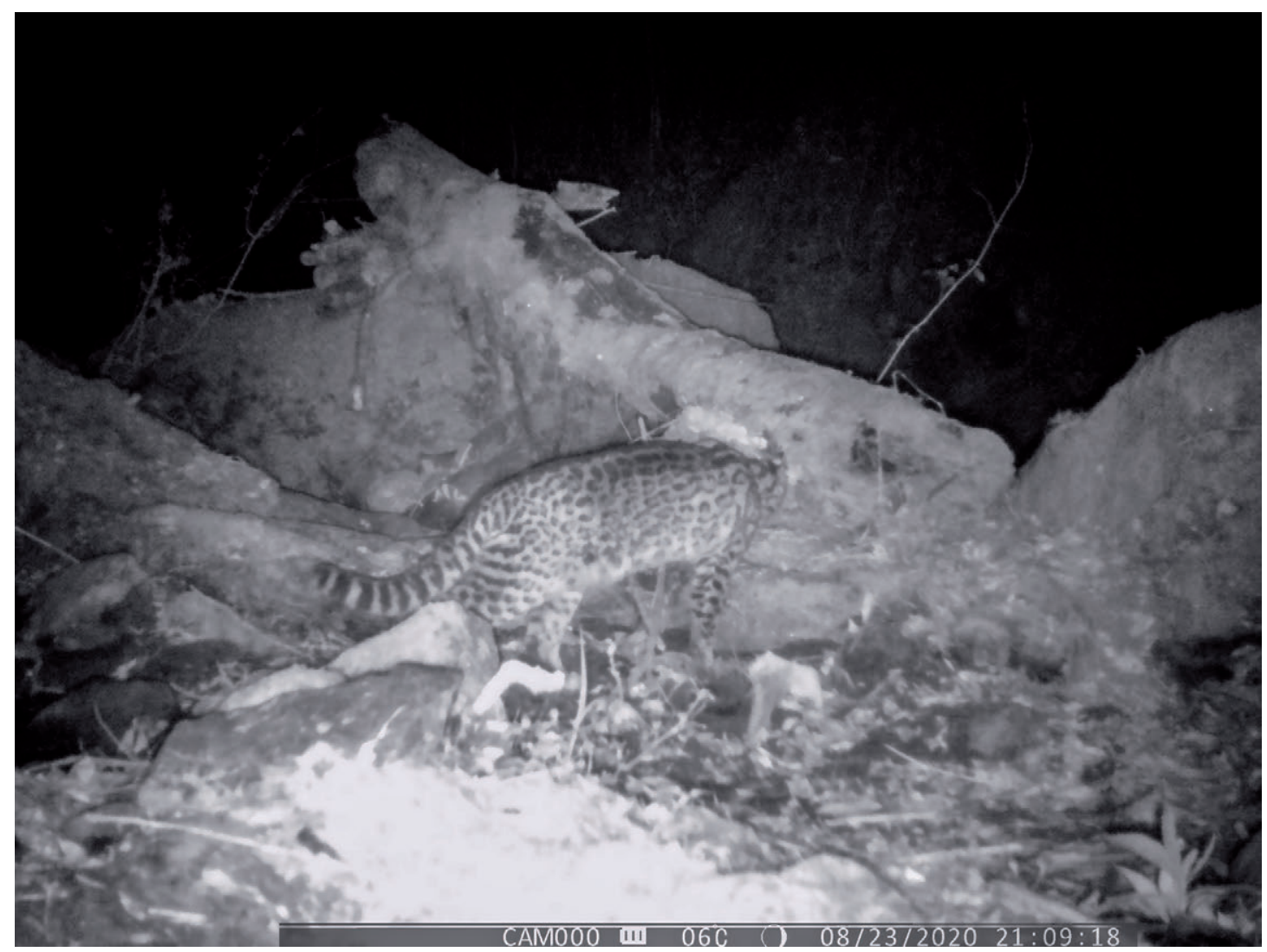

Figura 3. Fotografía perteneciente al segundo registro, el día 23 de agosto de 2020. En la imagen se observan los rasgos característicos de L. pardalis, como su patrón de manchas y su cola corta y ancha (Chebez, 1994; Canevari y Fernández Balboa, 2003).

Figure 3. Photo belonging to the second record, on August 23, 2020. The image shows the characteristic features of $L$. pardalis, such as its spots pattern and its short and wide tail (Chebez, 1994; Canevari y Fernández Balboa, 2003).

años posteriores pasó a ser dudosa debido a la posible extinción local de la especie (Cruz et al., 2019).

La conservación del bosque nativo será indispensable para su reproducción. Las principales amenazas que enfrenta esta especie son la degradación de su hábitat y la caza furtiva (Clavijo y Ramírez, 2009; Parera, 2018; Cruz et al., 2019). Sin embargo, aún hay grandes áreas con selva y bosque en muy buen estado de conservación que deberían ser protegidas y que solamente gracias a su fuerte pendiente o terreno escarpado no han sido modificadas por la actividad humana, como es el caso del sitio estudiado en el presente trabajo.

Con la confirmación de la presencia de este felino en la provincia de Catamarca es imprescindible tomar medidas efectivas de gestión y conservación, como la creación de áreas naturales protegidas estrictas para preservar las poblaciones más australes de $L$. pardalis en las Yungas. De igual manera, es sumamente importante realizar estudios futuros para conocer su distribución y población de esta especie en toda la provincia. 


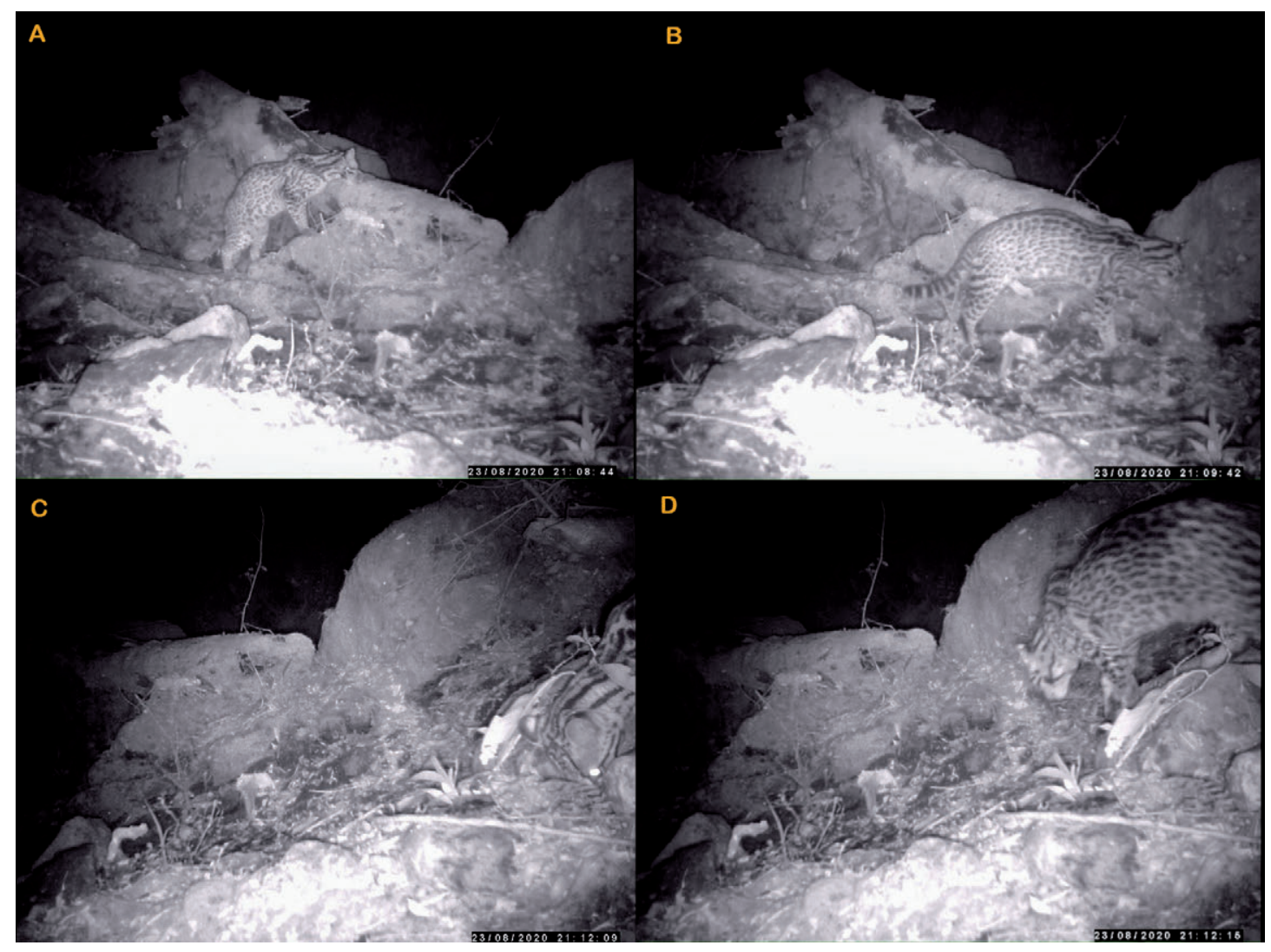

Figura 4. Capturas de los videos en los que fue registrado L. pardalis el día 23 de agosto de 2020. En "B" se logra ver de forma muy clara el patrón de manchas de su cuerpo.

Figure 4. Captures of the videos in which L. pardalis was recorded on August 23, 2020. In "B" it is very clearly the pattern of spots on its body.

\section{AGRADECIMIENTOS}

A César Hernán Barrionuevo, Viviana Martínez y Carolina Corpacci por su colaboración y acompañamiento con las tareas a campo. A Aníbal Fernando Parera, por la colaboración con bibliografía. Agradezco también al editor asociado de Acta Zoológica Lilloana y a los dos revisores por sus valiosas contribuciones que mejoraron sustancialmente la calidad del manuscrito.

\section{FINANCIAMIENTO}

Este trabajo no contó con financiamiento institucional.

\section{PARTICIPACIÓN}

Gonzalo Martínez: Tarea de campo, colocación de cámaras de fototrampeo, edición de la cartografía y redacción del manuscrito. 


\section{CONFLICTOS DE INTERÉS}

El autor declara que no existen conflictos de interés con otros autores ni con terceros.

\section{LITERATURA CITADA}

Administración de Parques Nacionales (APN). (2020). Leopardus pardalis: Sistema de Información de Biodiversidad (SIB). https://sib.gob.ar/especies/leoparduspardalis.

Barquez, R. M., Díaz, M. M., Ojeda, R. A. (2006). Mamíferos de Argentina: sistemática y distribución. Argentina: Sociedad Argentina para el Estudio de los Mamíferos (SAREM).

Brown, A., Martínez Ortiz, U., Acerbi, M., Corcuera, J. F. (2006). La situación ambiental Argentina 2005. Fundación Vida Silvestre Argentina, Buenos Aires.

Cabrera, A., (1957). Catálogo de los mamíferos de América del Sur. Revista del Museo Argentino de Ciencias Naturales "Bemandino Rivadavia" e Instituto Nacional de Investigación de las Ciencias Naturales, Ciencias Zoológicas, 4, 1-307.

Cavenari, M., Fernández Balboa, C. (2003). 100 mamíferos argentinos. Primera Edición. Buenos Aires: Albatros Ediciones.

Caziani, S., Trucco, C.E., Perovic, P., Tálamo, A., Derlindati, E., Dámoli, J., Lobo, F., Fabrezi, M., Srur, M., Quiroga, V, Martínez Oliver, M. I. (2003). Línea de base y programa de monitoreo de biodiversidad del Parque Nacional Copo. Proyecto de Conservación de la Biodiversidad- BIRF/GEF TF 028372-AR, Administración de Parques Nacionales, Argentina. 235 pp.

Chatellenaz, M. L., Villordo, M. G., Anchetti, J. L., Rolón, L. (2018). Primeros registros documentados del gato onza Leopardus pardalis (Carnivora: Felidae) en la provincia de Corrientes, Argentina. Acta Zoológica Lilloana, 62, 28-35. DOI: ht tps ://doi .org/10.30550/j .az 1/2018.62.1/3.

Chebez, J. C. (1994). Los que se van. Especies argentinas en peligro. Buenos Aires: Albatros.

Clavijo, A., Ramírez, G. F. (2009). Taxonomía, distribución y estado de conservación de los felinos sudamericanos: Revisión Monográfica. Boletín Científico Museo de Historia Natural, 13, 43-60.

Cruz, P., Varela, D., Di Bitetti, M. S., Paviolo, A., Quiroga, V. A., De Angelo, C., Cirignoli, S., De Bustos, S., Iezzi, M. E., Cuyckens, G. A. E., Albanesi, S., Aquino, J., Arrabal, J. P. (2019). Leopardus pardalis. En: SAyDS-SAREM (eds.) Categorización 2019 de los mamíferos de Argentina según su riesgo de extinción. Lista Roja de los mamíferos de Argentina. Versión digital: http://cma.sarem.org.ar.

Google Earth Pro. (2019). Imagen satelital de la provincia de Catamarca. Descargado el 8 de octubre de 2019.

Instituto Geográfico Nacional (IGN). (2014). Mapa Satelital de la provincia de Catamarca: República Argentina. Mapa físico-político.

Jayat, J. P., Barquez, R. M., Díaz, M. M., Martínez, P. J. (1999). Aportes al conocimiento de la distribución de los carnívoros del noroeste de Argentina. Mastozoología Neotropical, 6, 15-30. 
Morlans, M.C. (1995). Regiones naturales de la Provincia de Catamarca: Provincias Geológicas y Provincias Fitogeográficas. Revista de Ciencia y Técnica, 2, 142.

Nanni, A. S., Piquer-Rodríguez, M., Rodríguez, D., Núñez-Regueiro, M., Periago, M. E., Aguiar, S., Ballari, S. A., Blundo, C., Derlindati, E., Di Blanco, Y., Eljall, A., Grau, R. H., Herrera, L., Huertas Herrera, A., Izquierdo, A. E., Lescano, J., Macchi, L., Mazzini, F., Milkovic, M., Montii, L., Paviolo, A., Pereyra, M., Quintana, R. D., Quiroga, V., Renison, D., Santos Beade, M., Schaaf, A., Gasparri, N. I. (2020). Presiones sobre la conservación asociadas al uso de la tierra en las ecorregiones terrestres de la Argentina. Ecología Austral, 30, 304-320. hÀÛ4Ýps://doi.org/10.25260/EA.20.30.2.0.1056.

Parera, A. F. (2018). Los mamíferos de la Argentina y la región austral de Sudamérica. Segunda Edición. Corrientes, Argentina: AP Ediciones Naturales.

Paviolo, A., Crawshaw, P., Caso, A., De Oliveira, T., Lopez-Gonzalez, C. A., Kelly, M., De Angelo, C., Payan, E. (2016). Leopardus pardalis. The IUCN Red List of threatened species 2016: e.T11509A97212355. Consultado en https://www.iucnredlist.org/species/11509/97212355. Consultado el 17 de diciembre de 2020.

Perea, M., Pedraza, G., Luceros, J. (2007). Relevamiento de flora arbórea autóctona en la Provincia de Catamarca. Dirección de Recursos Naturales. Secretaría del Agua y el Ambiente. Buenos Aires: Consejo Federal de Inversiones (CFI). http://biblioteca.cfi.org.ar/wp-content/uploads/sites/2/2007/01/45417.pdf.

Servicio Meteorológico Nacional (SMN). (2020). Atlas Climático Argentina. Consultado en https://www.smn.gob.ar/clima/atlasclimatico. Consultado el 17 de diciembre de 2020.

Sistema de Información de Biodiversidad (SIB). (2020). Avistajes de Leopardus pardalis en Argentina. https://sib.gob.ar/especies/leopardus-pardalis?tab=avistajes. Consultado el 10 de julio de 2020.

Unión Internacional para la Conservación de la Naturaleza (UICN). (2020). La Lista Roja de especies amenazadas de la UICN. Versión 2020-2. https://www.iucnredlist.org. Consultado el 09 de julio de 2020. 